\title{
A new Philippine geoid model from airborne and terrestrial gravity data
}

\author{
Ronaldo Cruz Gatchalian ${ }^{1, *}$, René Forsberg ${ }^{2}$, and Arne Vestergaard Olesen ${ }^{3}$ \\ ${ }^{1}$ National Mapping and Resource Information Authority, Taguig City, Philippines \\ ${ }^{2}$ National Space Institute, Technical University of Denmark, Denmark \\ ${ }^{3}$ WGES, Denmark
}

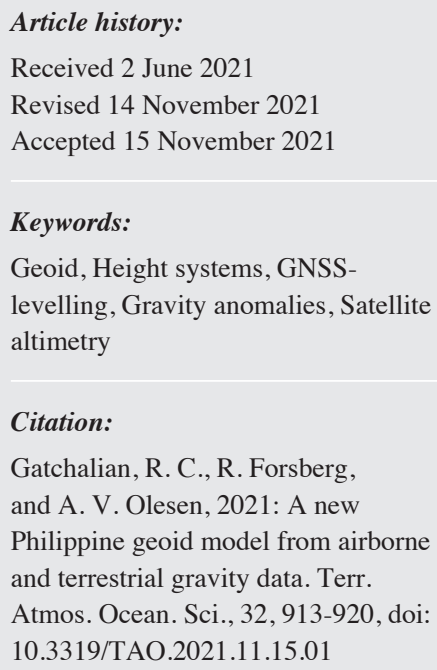

\begin{abstract}
This paper discusses the computation and re-computations of the preliminary Philippine Geoid Model 2014 (PGM2014) into PGM2016 and then PGM2018 with the technical assistance of the Technical University of Denmark (DTU-Space) using data from land gravity, airborne gravity, marine satellite altimetry and satellite gravity data from the ESA GOCE mission. Digital terrain models used in the computation process were based on 15" SRTM data, and all data combined with remove-restore state-of-the art geoid determination methods. The preliminary 2014 geoid model was computed in a global vertical reference system with an estimated standard deviation around $0.25 \mathrm{~m}$, then fitted to GNSS/Leveling with an a priori RMS value of only $0.50 \mathrm{~m}$ at that point in time, highlighting the challenges in the geodetic infrastructure of the Philippines, with extreme gravity field variations, and geodynamics which can make GNSS-levelling comparison geoid values highly error-prone. To improve the PGM2014, an interim PGM2016 hybrid geoid was computed using reprocessed, reanalyzed and densified land gravity and improved GNSS/Leveling data. Significant improvement was seen in the reprocessed gravity data as well as the final geoid (SD $=0.022 \mathrm{~m} ; \mathrm{RMS}=0.054 \mathrm{~m}$ ) after a more tight GNSS fit, taking into account the different vertical datum used in the islands. In 2018, with new satellite data available, densified gravity data, and additional GNSS/Leveling points were used in a new geoid computation. The new computed geoid has an improved fit to GNSS/Leveling of $2 \mathrm{~cm}$. The formal error estimate of the new hybrid geoid across the Philippines is around $10 \mathrm{~cm} \mathrm{rms}$, still limited to some degree by island height system and geodynamics effects.
\end{abstract}

\section{INTRODUCTION}

In almost all projects of building "something" on the surface of the earth, there is a need to determine where the water will flow for the design of water supply and drainage systems. Therefore, elevation information of the area is a requirement of every project. This elevation information would require the determination of elevation of points on the ground. The conventional way of determining elevation of points is through the conduct of geodetic leveling, which is very tedious and time consuming. With the advent of GNSS, it has become possible to estimate mean sea level (MSL) elevation of points using the formula

\footnotetext{
* Corresponding author

E-mail:rcgatchalian@namria.gov.ph
}

$H=h-N$

where $H$, height referred to MSL, $h$ is the ellipsoidal height from GNSS survey, and $N$ is the geoid height.

The geoid height can be supplied by a geoid model, computed from gravity data, while $h$ can be obtained by GNSS. Using a geoid model together with GNSS will save time and money with projects that do not need very high elevation accuracy. Computing a geoid model for the Philippines has always been the aspiration of the Geodesy Division of the National Mapping and Resource Information Authority (NAMRIA). This paper will briefly describe the computation of the recent (Forsberg et al. 2014) Philippine Geoid Model (PGM2014), its airborne gravity survey, land gravity data, and the geoid nominal accuracies. The third 
part will talk about the re-computation and improvement of the preliminary geoid into PGM2016. Section 4 will discuss the new satellite gravity data, additional land gravity and GNSS/Levelling data used in the new 2018 computation. All computations include quality control plots of the land gravity and GNSS/leveling fit.

\section{THE PRELIMINARY PGM2014}

In 2014, the National Space Institute of the Denmark Technical University (DTU-Space) conducted a nationwide airborne gravity survey funded by the National Geospatial Intelligence Agency (NGA) to improve the global gravity field model EGM2008. The mean altitude for all flights was $3185 \mathrm{~m}$ with a terrain clearance of $545 \mathrm{~m}$ above mountains and $3760 \mathrm{~m}$ in lowlands as shown in the flight tracks. The estimated rms error of the airborne data is $2.6 \mathrm{mGal}$, based on cross-over error analysis; since tracks were flown at nonconstant heights (Fig. 1), the real accuracy might be better.

Following the airborne gravity survey, the NAMRIA has computed a preliminary hybrid geoid model - Philippine Geoid Model 2014 (PGM2014) with the assistance of Professor Rene Forsberg. PGM2014 used data from 1261 land gravity points, the airborne gravity survey, marine satellite altimetry (DTU-10), and the newest satellite gravity data from the Gravity Field and Steady-State Ocean Circulation Explorer (GOCE) mission release 5. The geoid was computed using RTM terrain reductions, and a rigorous downward continuation process by least-squares collocation using the GRAVSOFT Package, a set of Fortran routines developed through many years of research and project work at DTU-Space and Niels Bohr Institute, University of Copenhagen (Forsberg and Tscherning 2008). The details of the method are described in detail in (Forsberg et al. 2014). Comparisons of airborne and land gravity data after terrain and EGM reduction were done for quality control. Large errors in the land gravity data were discovered (air-land differences of $>50 \mathrm{mGal}$ ) and are shown in Fig. 2. The final geoid "restore" statistics and standard deviations (SD's) of the reduced geoid are listed in Table 1.

For a hybrid or MSL-based geoid, a set of 190 GNSS data in ITRF2005 at levelling benchmarks was made to fit the gravimetric geoid. This is done by "forcing" the "gravimetric" geoid heights $\left(N_{\text {grav }}\right)$, computed in a world height system, to coincide with the observed geoid heights of GNSS/levelling $\left(N_{h-H}\right)$, thus preserving the existing vertical datum of the country, i.e., MSL. In this fitting, these GNSS data showed a relatively large error relative to the geoid, with large outliers in some regions, likely due to a combination of geodynamic effects and levelling and/or GNSS errors, as well as the separate tide-gauge defined height systems on different islands.

The rms fit of GNSS/levelling was $0.5 \mathrm{~m}$ with maxi- mum offset value of $1.49 \mathrm{~m}$; it is therefore not possible to use these data for validation of the geoid. Figure 3 shows the offset values, and the geoid correction surface for a fitted geoid (the "corrector" surface was gridded with $80 \mathrm{~km}$ correlation length, and GNSS-Levelling apriori error of $10 \mathrm{~cm}$ ). Figure 4 shows the comparison of PGM2014 (gravimetric) to EGM2008; large improvements are seen, especially in the south.

\subsection{RE-COMPUTATION OF THE GEOID}

With large errors in land gravity and GNSS/Levelling data apparent in PGM2014, this geoid model will not satisfy most GNSS survey needs requiring accuracies of $10 \mathrm{~cm}$ or better. Although the PGM2014 can be used in topographic surveys in remote areas where a rough elevation estimate of less than $1 \mathrm{~m}$ will suffice, this geoid can still and should be improved. Naturally, all surveyors would want a geoid model that can produce elevations that are a little less accurate than levelling, say $<10 \mathrm{~cm}$ Standard Deviation (SD) at $95 \%$ confidence level. To further improve a geoid model, we followed common geodetic advice, as outlined in the paper "Towards a cm-geoid in Malaysia" (Forsberg 2005):

- Levelling networks must be carefully analysed for adjustment errors;

- Connections and antenna height errors of GPS data on benchmarks must also be revisited and re-analysed;

- Erroneous points (geoid outliers) must be resurveyed by Levelling and GPS;

- New GPS-fitted version of the geoid must be computed as new batches of GPS-Levelling data, additional gravity surveys in major cities and GPS user's height problem reports comes in.

In 2015, with the help of Professor Forsberg, NAMRIA started the re-computation of the PGM2014 (Gatchalian et al. 2016). In this re-computation, the 2014 satellite and airborne data processing results were used; only the land gravity, GNSS and Levelling data were reprocessed and re-analysed.

\subsection{Land Gravity 2016}

The 1261 land gravity data was reviewed and reprocessed. Errors in position of the land gravity data "outliers" in the 2014 computation were discovered, corrected and reprocessed. Densification of land gravity stations was also conducted in some major cities of the country, bringing the number to 2214 points. One quality check of the land gravity data is the comparison of its anomalies with that of the airborne, see Fig. 5; this is done after the subtraction of terrain effects in the rough mountains. Significant improvements can be seen in the land data. Most dots are in green, some yellows and light blue $(35-50 \mathrm{mGal}$ difference in 
mountainous areas only). This highlights the need for careful QC of terrestrial gravity anomaly data, and how new airborne data can help to find systematic errors in older data.

\subsection{GNSS/Levelling Data 2016 and PGM2016}

The 2016 levelling data was re-analysed, readjusted and corrected, with some outliers deleted. The GNSS data was reprocessed and readjusted, points with large height errors and ellipses were deleted. After removing the GNSS/ Levelling outliers, 101 out of the 190 BMs remain and used in fitting to the re-computed gravimetric geoid. After fitting the new GNSS/Levelling, the RMS fit is now $0.054 \mathrm{~m}$ with a minimum and maximum offset value of -0.124 and $0.169 \mathrm{~m}$ respectively. This improvement is mainly due to the removal of erroneous levelling and GNSS points, considering there are also unknown datum differences on many smaller islands. Figure 6 shows the offset values and the new geoid

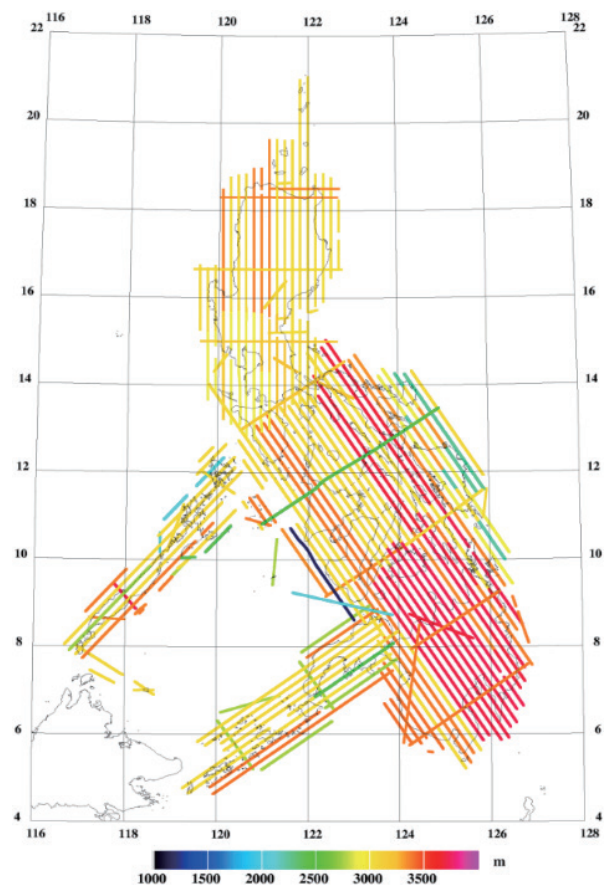

Fig. 1. Color-coded flight track elevations of the DTU/NGA/NAMRIA airborne gravity survey, 2014. The varying flight elevations, due to terrain and aviation restrictions, are handled efficiently in the downward continuation and gridding process. correction surface of PGM2016.

The PGM2014 was re-computed to PGM2016 (using the improved land gravity data) with a SD of $0.022 \mathrm{~m}$ as shown in Table 2. This represented a major improvement in the geoid for GNSS use, and again highlighted the need for careful QC of all data. Only the uniform-quality airborne data were left unchanged for PGM2016.

\section{2018 COMPUTATION OF THE GEOID}

In 2018, with the availability of new satellite data, the Philippine geoid model was recomputed again with the original airborne, additional land gravity and GNSS/Levelling data. A new global model PGM2017 (preliminary gravitational model 2017, a precursor of the new EGM2020 model) and updated DTU15 satellite data were used in this re-computation. The shift to the reference model PGM2017 was important, as this model also contains the airborne

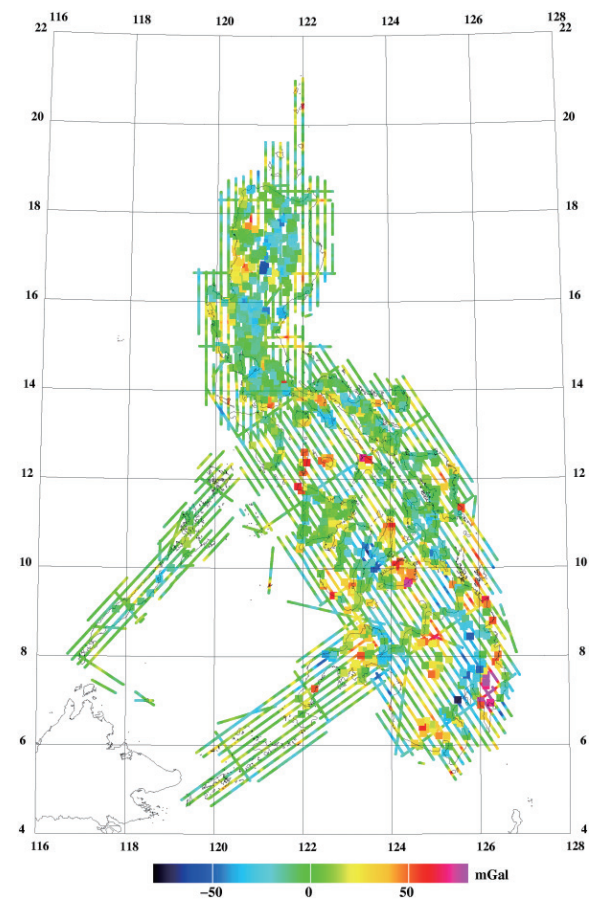

Fig. 2. Outliers of the 2014 quality check between land and airborne terrain-corrected gravity data (magenta and dark blue dots).

Table 1. Computed geoid statistics and standard deviation of PGM2014.

\begin{tabular}{c|cccc}
\hline Unit: Meters & Mean & Std. dev. & Min & Max \\
\hline Reduced geoid & 0.00 & 0.25 & -1.61 & 2.88 \\
RTM restore effects & 0.00 & 0.04 & -0.23 & 0.74 \\
Final gravimetric geoid & 39.06 & 18.36 & -9.02 & 76.43 \\
\hline
\end{tabular}




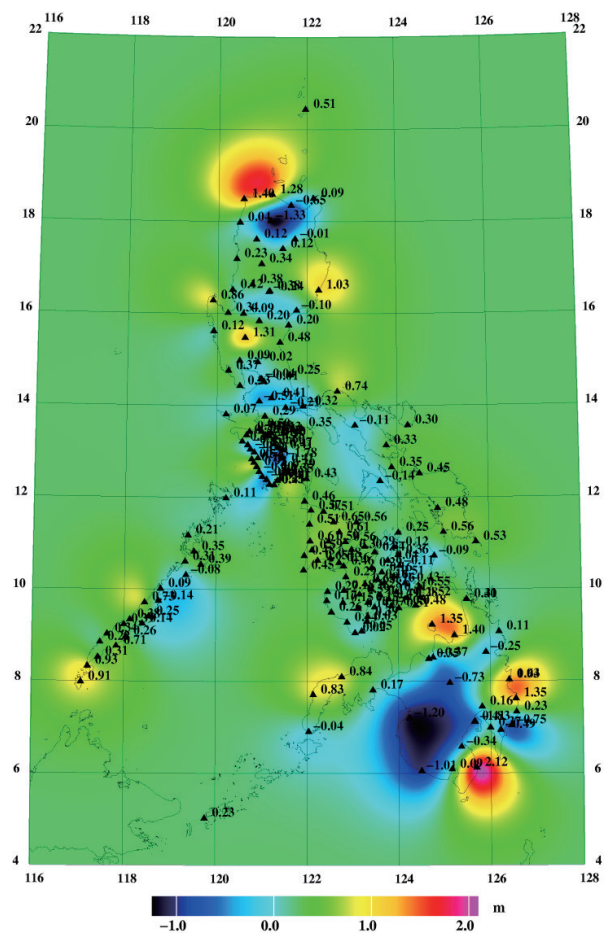

Fig. 3. Location of GNSS/Leveling data in PGM2014. Color shows the offset values of the fitted geoid, after a gridding by least-squares collocation/Kriging.

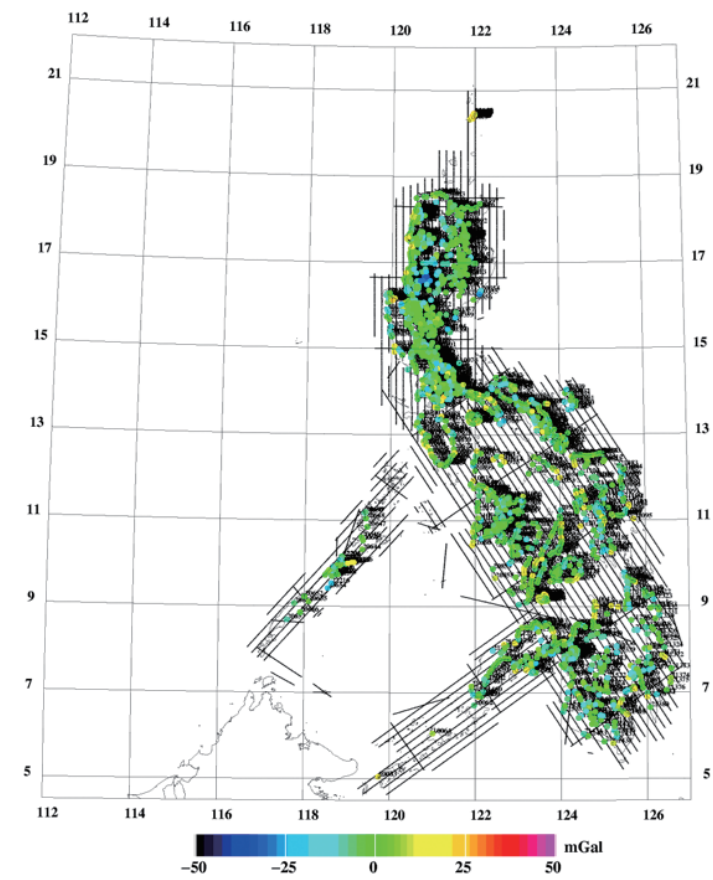

Fig. 5. PGM2016 land gravity data after reprocessing, plotted as difference to airborne data. Most differences are below $25 \mathrm{mGal}$, some points exceed $35 \mathrm{mGal}$ in mountainous regions, which is sufficient given the inherent filtering of airborne data.

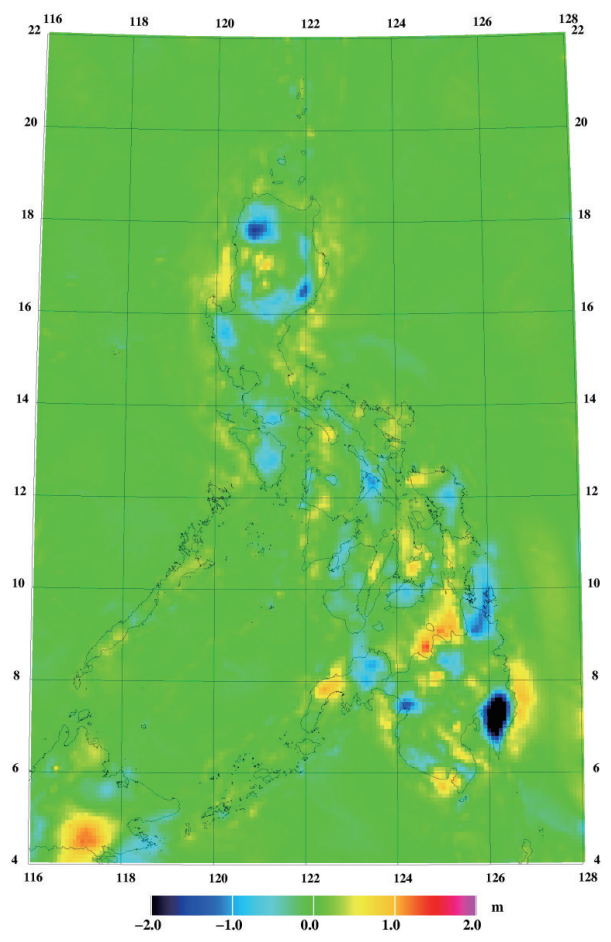

Fig. 4. Differences between geoid heights (Gravimetric) in the PGM and EGM2008. The large differences illustrate the large improvements in the reference model by the inclusion of the new airborne data.

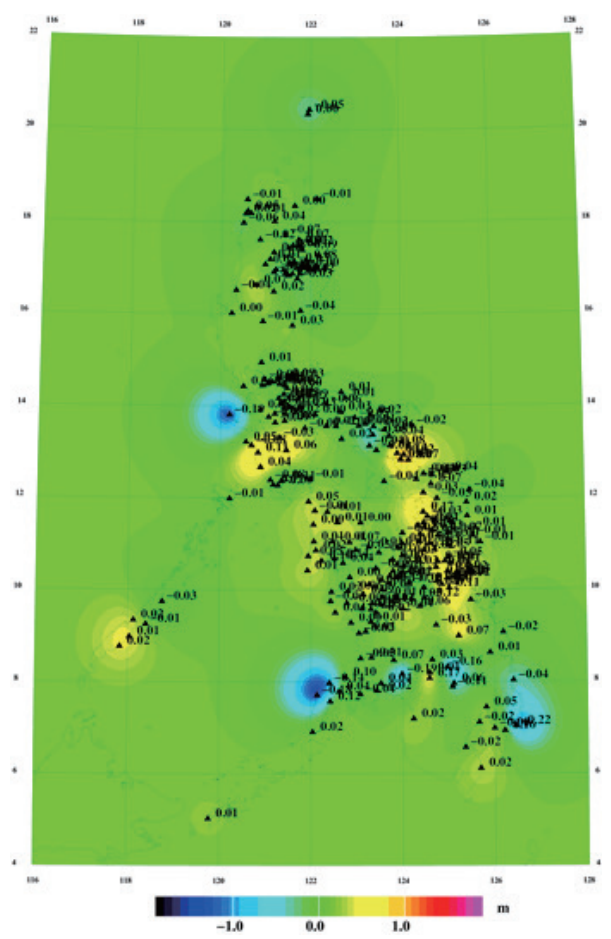

Fig. 6. New offset values of the fitted PGM2016. A few outliers are still seen, but source of these are difficult to assess. 
Table 2. Computed geoid statistics and standard deviation of PGM2016.

\begin{tabular}{c|cccc}
\hline Unit: Meters & Mean & Std. dev. & Min & Max \\
\hline Reduced geoid & 0.00 & 0.022 & -0.16 & 0.44 \\
RTM restore effects & 0.00 & 0.25 & -1.69 & 3.12 \\
Final gravimetric geoid & 38.26 & 18.36 & -9.82 & 76.63 \\
\hline
\end{tabular}

Phillipines data from 2014.

\subsection{Land Gravity Data}

5779 land gravity data points were gathered, processed and tabulated in excel format: ID, Long, Lat, Gravity Value, Elevation - as required by the anomaly job program. Figure 7 shows the distribution of the densified land gravity data in the form of a Bouguer anomaly plot. The color-coded dots seem to conform to each other, indicating that the gravity data seems now bias and error free. For quality checking, the air-land Bouguer anomaly differences range up to $18.4 \mathrm{mGal}$, with a mean of $0.01 \mathrm{mGal}$, using only close-by gravity points near the airborne data positions, as shown in Fig. 8. More land gravity data, up to 41000 points, will be added from 2019 until 2030 in order to further refine and re-compute a new version and the Philippine geoid.

\subsection{GNSS/Levelling Data}

A set of $286 \mathrm{GNSS} /$ Levelling data were used in fitting the PGM2018 geoid - some coming from the 2016 data (101pts) then supplemented by new GNSS surveys. Figure 9 shows the distribution of Benchmarks (BMs) surveyed by GNSS. The survey was done from 2010 until 2018 with the additional points coming from the PGM Validation project that started in 2016. The GNSS survey observation time range from 2 to $6 \mathrm{hrs}$ depending on the length of the baseline. GNSS ellipsoid height errors after adjustment range from 0.013 to $0.094 \mathrm{~m}$, while planar error ellipses are less than $0.05 \mathrm{~m}$.

The levelling data points came from the adjusted First Order Level networks nationwide with 1.96-sigma SD ranging from 0.005 to $0.05 \mathrm{~m}$. The BMs were selected according to their location (with clear view of the sky) for the consideration of the GNSS survey requirements. GNSS/Levelling data format is: ID, Lat, Long, Elevation, $N_{h-H}$, for use of the fitgeoid job program.

The RMS value of the geoid fit was $0.022 \mathrm{~m}$ with minimum and maximum offset values of -0.058 and $0.063 \mathrm{~m}$ respectively, see Table 3. Figure 10 shows the post-fit offset values, and the geoid correction surface for a fitted geoid (the corrector surface was gridded with least squares collocation, with $60 \mathrm{~km}$ correlation length, and a GNSS-
Levelling apriori error of $10 \mathrm{~cm}$ ). More points will be added to the GNSS/Levelling data as the PGM Validation Survey progresses.

It should be noted that in the fitting process the post-fit accuracy is very dependent on the a priori SD assumptions of GNSS and levelling accuracy, as well as the correlation length of the fit. To judge the accuracy of the underlying pre-fit gravimetric geoid accuracy, Fig. 11 shows the error estimates of the predicted geoid, with only one GNSS point - the Primary MSL fundamental tide gauge in Manila - fixed. Because of the memory restrictions of a single collocation error estimation run, all data have been selected only at $0.1^{\circ}$ resolution $\left(0.2^{\circ}\right.$ for DTU15). The data shows that the gravimetric geoid has an accuracy of around $10 \mathrm{~cm}$ across most of the Philippines territory; it should be noted, though, that the underlying PGM2018 gravimetric geoid is in a global reference system, and not fitted across the various height datums on the islands.

\subsection{The Philippine Geoid 2018}

Repeating the steps enumerated in the PGM2014 computation, the Philippine 2018 geoid has been computed using PGM2017, DTU15, airborne, and land gravity data. The accuracy is now $0.01 \mathrm{~m}$ as listed in Table 4 , and the underlying gravimetric geoid better than $10 \mathrm{~cm}$.

Figure 12 shows the new PGM2018. The geoid model file (in GRAVSOFT.gri, Trimble.ggf, or geotiff formats), along with user-friendly interpolation software, is available for download at the NAMRIA website, https://www.namria.gov.ph.

\section{CONCLUSION}

With the sequence of geoids (PGM2014, PGM2016, and PGM2018), it is shown that careful QC and new data acquisition have generated an accurate new geoid of the Philippines, one of the most challenging areas of the world to do this, due to the large gravity anomaly variations, and major geodynamics and subsidence areas.

The computation and re-computation of the Philippine Geoid Model has been described, from the preliminary PGM2014 model, its re-computation to PGM2016, and then to the present PGM2018. The nominal accuracy of the PGM2018 is about $3 \mathrm{~cm}$ (after the fit to GNSS/levelling). 


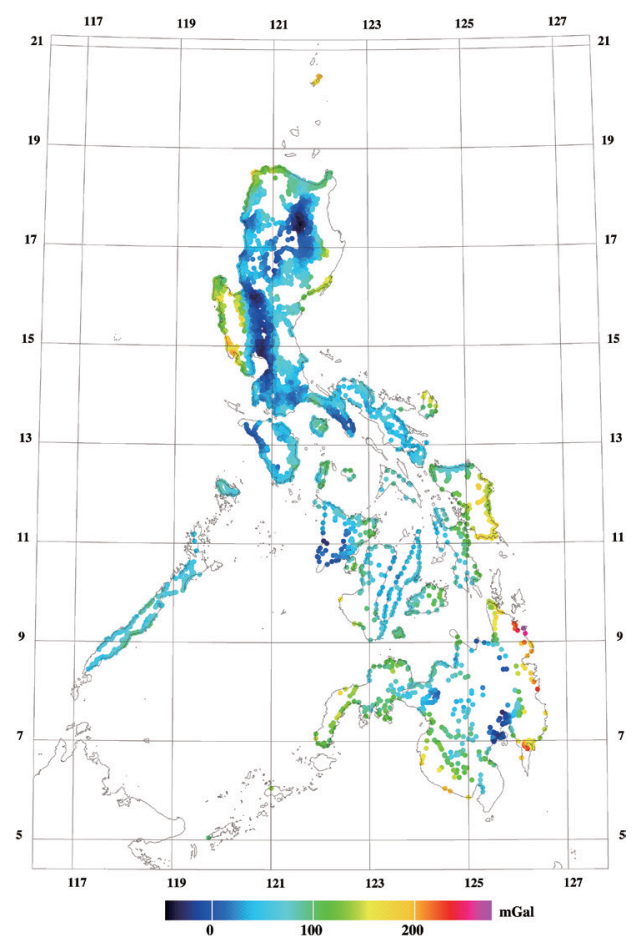

Fig. 7. Distribution of land gravity points used in PGM2018.

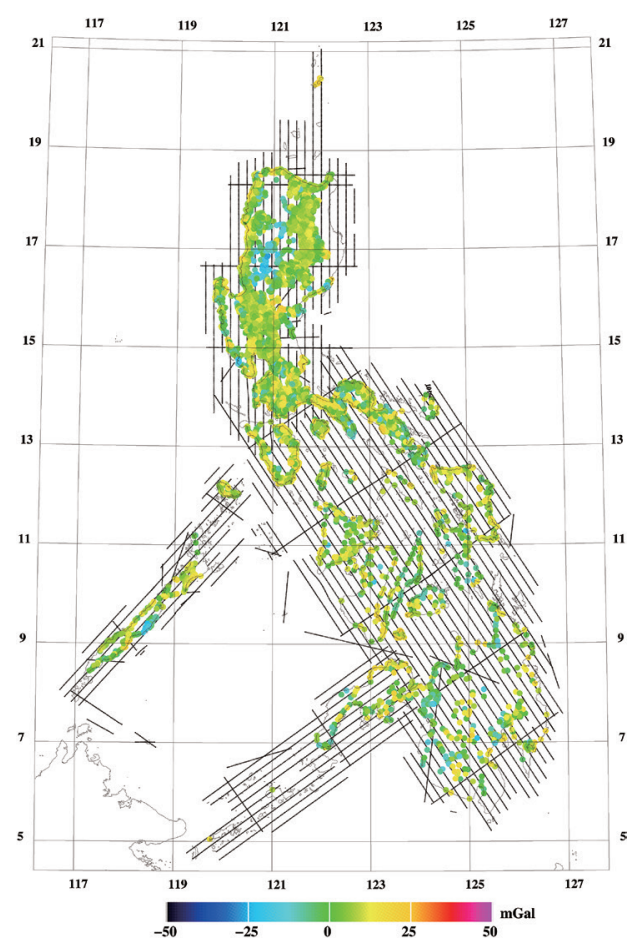

Fig. 8. Differences between airborne and land anomalies for the PGM2018 geoid.

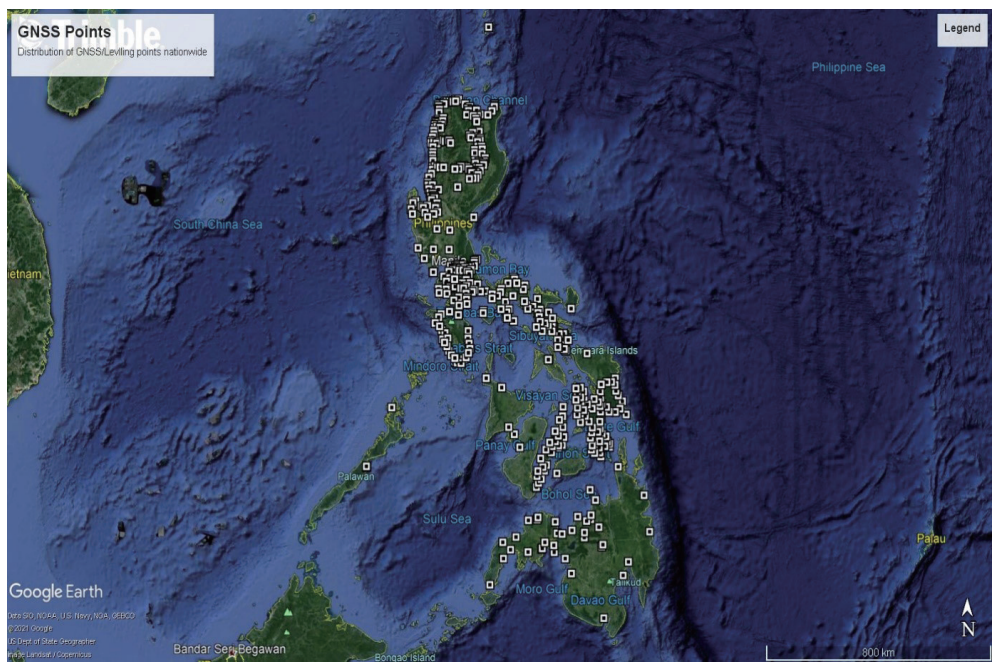

Fig. 9. The GNSS surveyed BMs as of 2018.

Table 3. Fitting statistics before and after fitgeoid job program (PGM2018).

\begin{tabular}{c|cccc}
\hline Unit: Meters & Mean & Std. dev. & Min & Max \\
\hline Before Fit & 1.653 & 0.531 & 0.422 & 2.737 \\
After Fit & 0.001 & 0.022 & -0.58 & 0.63 \\
\hline
\end{tabular}




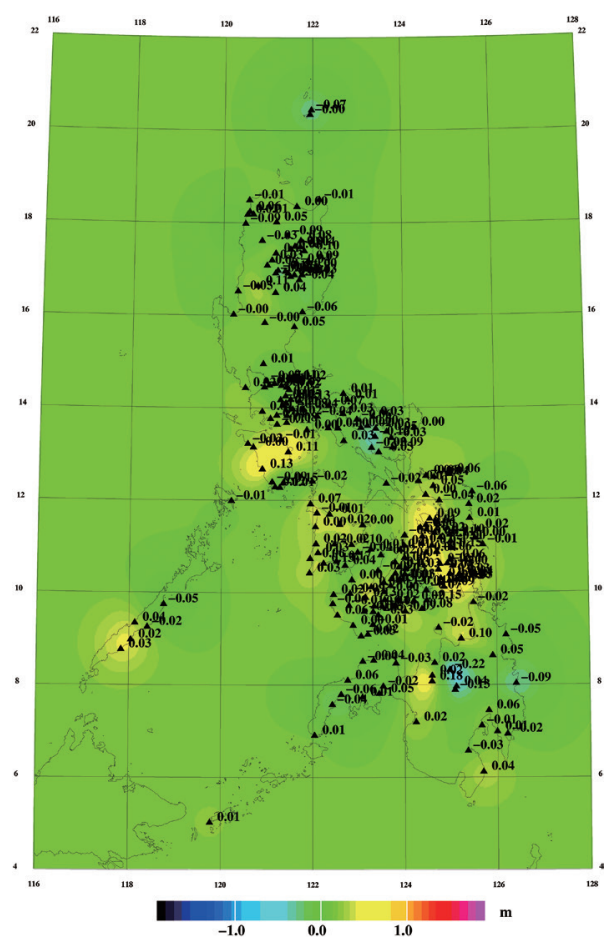

Fig. 10. PGM2018 GNSS/Levelling offset values. Most points are colored green and yellow indicating a better fit of the geoid to the BMs.

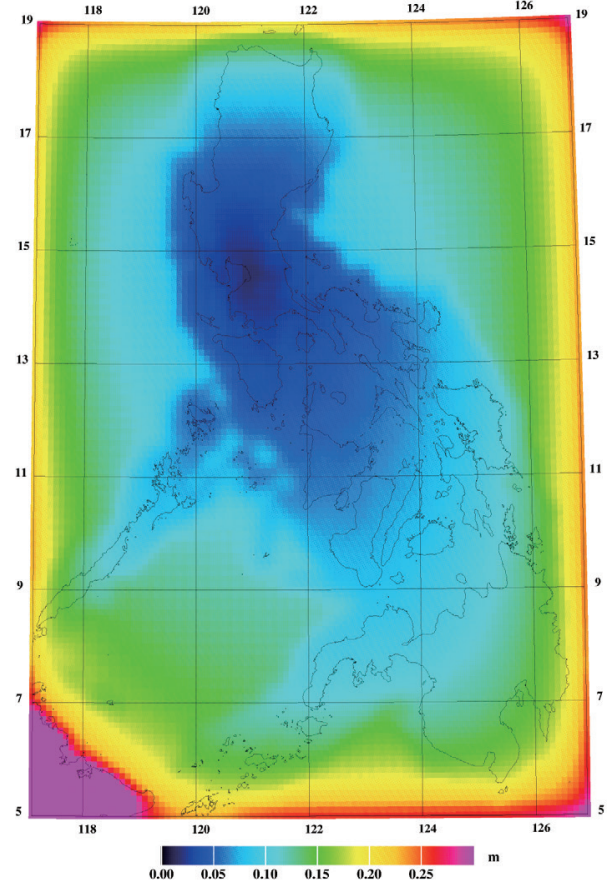

Fig. 11. Errors of the underlying gravimetric geoid, relative to the Manila reference tide gauge. Errors only computed assuming data in the shown region, with a covariance model fitted to the actual data thinned to $0.1^{\circ}$ resolution.

Table 4. Computed geoid statistics and standard deviation of PGM2018.

\begin{tabular}{c|cccc}
\hline Unit: Meters & Mean & Std. dev. & Min & Max \\
\hline Reduced geoid & 0.00 & 0.01 & -0.16 & 0.44 \\
RTM restore effects & 0.00 & 0.25 & -1.67 & 3.13 \\
Final gravimetric geoid & 38.95 & 18.36 & -9.14 & 76.32 \\
\hline
\end{tabular}

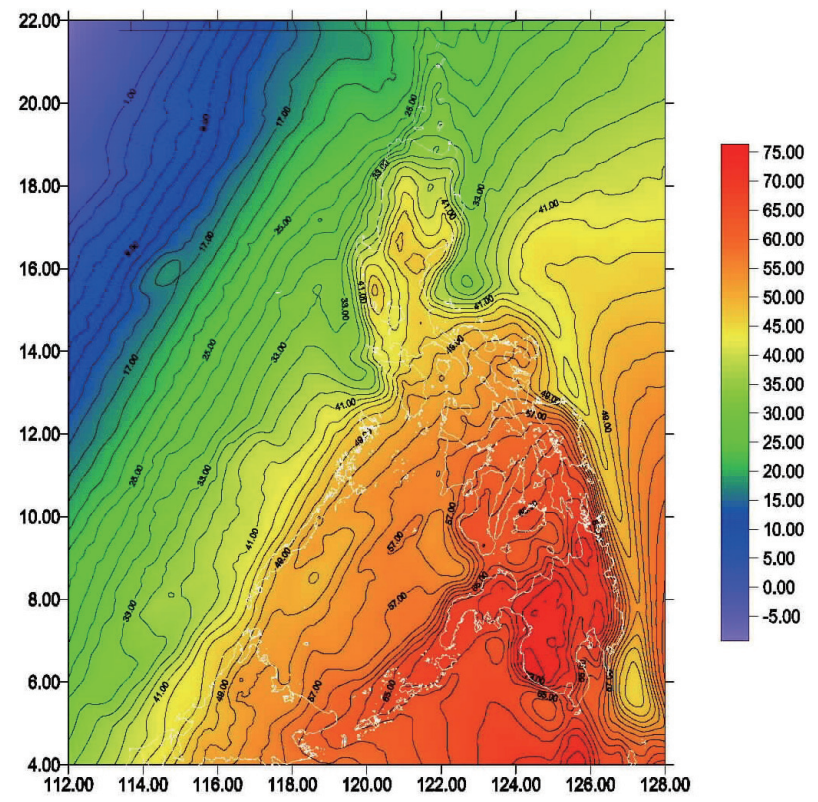

Fig. 12. The new PGM2018 with contour interval $2 \mathrm{~m}$. 
To preserve the existing vertical reference datum of the topographic maps, the computed geoid was reduced to the benchmark ML-3 reference level surface to roughly fit the geoid in Metro Manila area. Then, in order to close the gap between the MSLs and the geoid, and fit the latter to the different MSL reference level surfaces of the islands in the country, (which in effect unifies them into an equipotential surface) Tidal Benchmarks (TGBMs) and BMs nationwide were surveyed by GNSS. The SD of the fit is about $2 \mathrm{~cm}$. For GNSS survey projects requiring elevation accuracies of about 5 to $30 \mathrm{~cm}$, this geoid model is a good alternative to geodetic leveling. The resulting $\mathrm{H}$ can only be as accurate as the geoid model and the GNSS surveys, thus, the following should be noted:

(1) 3D coordinates of Ground Control Points change with time due to advancement in GNSS technology and crustal deformation.

(2) Ellipsoidal heights must be accurate, acquired and computed in about the same epoch as the GNSS/Leveling (2010 or later); if not, use a vertical deformation model to bring the coordinates to the correct epoch.

(3) If there is no vertical deformation model, obtain the updated coordinates by connecting to an updated (re-observed) geodetic control.

Further densification of the land gravity in towns and cities to 41000 points will be conducted from 2019 to 2030 to further refine the geoid. Re-computation will be done for the new version of the geoid as new gravity data and more GNSS/levelling data comes in.

\section{REFERENCES}

Forsberg, R., 2005: Towards a cm-geoid for Malaysia. Workshop on National RTK Network and Geoid Launch, Kuala Lumpur, Malaysia. Available at http:// ftp.spacecenter.dk/pub/malaysia.

Forsberg, R. and C. C. Tscherning, 2008: An overview manual for the GRAVSOFT: Geodetic Gravity Field Modelling Programs. DTU Space, 75 pp.

Forsberg, R., A. V. Olesen, R. Gatchalian, and C. C. C. Ortiz, 2014: Geoid model of the Philippines from airborne and surface gravity. National Mapping and Resource Information Authority (NAMRIA), Department of Environmental and Natural Resources, Republic of The Philippines, $12 \mathrm{pp}$. Available at https://www.isgeoid. polimi.it/Geoid/Asia/Philippines/ph geoid report.pdf.

Gatchalian,R., R. Forsberg, and A. Olesen, 2016: PGM2016: A new geoid model for the Philippines. Coordinates, XIII, 31-39. Available at https://mycoordinates.org/ pgm2016-a-new-geoid-model-for-the-philippines. 